\title{
Large-scale neural correlates of affective picture processing
}

\author{
ANDREAS KEIL, ${ }^{\mathrm{a}}$ MARGARET M. BRADLEY, ${ }^{\mathrm{b}}$ OLAF HAUK, ${ }^{\mathrm{c}}$ BRIGITTE ROCKSTROH, \\ THOMAS ELBERT, ${ }^{\mathrm{a}}$ AND PETER J. LANG ${ }^{\mathrm{b}}$ \\ ${ }^{a}$ Department of Psychology, University of Konstanz, Konstanz, Germany \\ ${ }^{\mathrm{b}}$ NIMH Center for the Study of Emotion and Attention, University of Florida, Gainesville, Florida, USA \\ ${ }^{\mathrm{c}}$ Medical Research Council, Cognition and Brain Sciences Unit, Cambridge, United Kingdom
}

\begin{abstract}
Hemodynamic and electrophysiological studies indicate differential brain response to emotionally arousing, compared to neutral, pictures. The time course and source distribution of electrocortical potentials in response to emotional stimuli, using a high-density electrode (129-sensor) array were examined here. Event-related potentials (ERPs) were recorded while participants viewed pleasant, neutral, and unpleasant pictures. ERP voltages were examined in six time intervals, roughly corresponding to P1, N1, early P3, late P3 and a slow wave window. Differential activity was found for emotional, compared to neutral, pictures at both of the P3 intervals, as well as enhancement of later posterior positivity. Source space projection was performed using a minimum norm procedure that estimates the source currents generating the extracranially measured electrical gradient. Sources of slow wave modulation were located in occipital and posterior parietal cortex, with a right-hemispheric dominance.
\end{abstract}

Descriptors: Dense-array electroencephalogram, Emotion, Motivation, Arousal, Picture perception, Source modeling, Minimum norm estimate

Viewing affective pictures elicits emotional reactions in selfreport, autonomic, and somatic measures (Bradley, Greenwald, Petry, \& Lang, 1992; Bradley, Lang, \& Cuthbert, 1993; Hamm, Greenwald, Bradley, \& Lang, 1993; Lang, Bradley, \& Cuthbert, 1990; Lang, Greenwald, Bradley, \& Hamm, 1993). Brain responses to affective pictures have been investigated using a variety of measures, including the event-related potential (ERP; Cacioppo \& Gardner, 1999; Cuthbert, Schupp, Bradley, Birbaumer, \& Lang, 2000). One finding of this research has been a modulation of late deflections of the ERP as a function of motivational significance (Lang, Bradley, \& Cuthbert, 1997). Specifically, greater magnitude of the P300 deflection as well as a sustained later positivity characterize the response to emotionally salient (i.e., pleasant or unpleasant) compared to neutral pictures (Cacioppo, Crites, Gardner, \& Bernston, 1994; Cuthbert et al., 2000; Keil et al., 2001; Laurian, Bader, Lanares, \& Oros, 1991; Mini, Palomba, Angrilli, \& Bravi, 1996; Palomba, Angrilli, \& Mini, 1997; Schupp et al., 2000). This effect has been theoretically related to motivated attention, in which motivationally relevant stimuli naturally and perhaps automatically arouse and direct attentional resources (Lang et al., 1997). Although these arousal-related signal enhancements have been found repeatedly, their neural origins are unclear, due, at

The authors thank Ursula Lommen and Klaus Lang for help in data acquisition.

Address reprint requests to: Andreas Keil, Department of Psychology, University of Konstanz, P.O. Box D25, D-78457 Konstanz, Germany. E-mail: Andreas.Keil@uni-konstanz.de. least in part, to the limited number of sensors available in standard electrode arrays and the resulting restriction of analyses to voltage maps.

Studies using functional magnetic resonance imaging (fMRI) have found more extensive activity in the visual cortex when viewing emotional compared to neutral pictures, with larger differences in the right, compared to left, hemisphere (e.g., Dolan et al., 1996; Lang et al., 1998). These data suggest that the visual cortex is differentially activated as a function of emotional arousal, and suggest that the larger positive electric potentials found for arousing compared to neutral pictures may be at least partially generated by differential activity in the visual cortex. Electrophysiological techniques could complement the hemodynamic data by providing additional information on the temporal characteristics of emotional picture processing.

In particular, differences in initial processing, reflected in early components of the ERP such as the P1/N1, may provide information that is not readily available using the slower hemodynamic measures. Several variables potentially involved in picture perception have been proposed to correlate with P1/N1 modulations. For example, Vogel and Luck (2000) have suggested that the visual N1 may be a correlate of a discrimination process. In addition, it has consistently been shown that paying attention to nonspatial object characteristics such as form or color modulates the ERP in the range of the visual $\mathrm{N} 1$, that is, around $150 \mathrm{~ms}$ after stimulus onset (Hillyard \& Anllo-Vento, 1998). In a similar manner, information regarding early stages of affective evaluation may be provided by analysis of early ERP parameters during affective tasks (Keil et al., 2001; Pizzagalli, Regard, \& Lehmann, 1999). 
In the present study, we used a high-density electrode array (129 sensors), together with a distributed source analysis procedure (minimum norm estimate), to investigate the temporal dynamics in picture perception and to estimate the cortical sources of changes in the scalp-recorded ERP. Distributed source modeling has been proposed as a means for enhancing the resolution of electrophysiological data without constraining the inferred electric activity to point sources or single dipoles (Hämäläinen \& Ilmoniemi, 1984). Consequently, a priori assumptions about the size, locations, and strength of a limited number of sources are not necessary. To obtain linear estimates of the electric sources, several procedures have been suggested, including computing the inverse solution on predefined surfaces (Knosche, Praamstra, Stegeman, \& Peters, 1996) or in a threedimensional volume (Pascual-Marqui, Michel, \& Lehmann, 1994). Using a linear estimation technique in a three-shell head model, we aimed at complementing the voltage data obtained using a densearray electroencephalogram (EEG) montage with information regarding their electric source distribution and strength to investigate the neural mechanisms underlying affective picture viewing. Although not providing precise anatomical localization, the minimum norm estimate of brain activity adds to the voltage data in several respects, including (a) the ability to infer the gross location of the origin of the signal, and (b) the representation of dipolar voltage gradients that are tangential with respect to the scalp at one focus of activity, rather than at two distant locations as is the case with voltage data (Hauk, Keil, Elbert, \& Müller, 2002).

In this study, subjects viewed a large sample of pleasant, neutral, and unpleasant pictures from the International Affective Picture System (IAPS; Center for the Study of Emotion and Attention, 1999). EEG was recorded from 129 electrodes during a 6-s pictureviewing interval. Based on previous ERP studies, we expected that emotional pictures would be associated with greater positivity, compared to neutral pictures, particularly in later time segments (>300 ms) after the onset of a picture. Furthermore, the sources of the signal contributing to differences in this late positive potential were predicted to lie within posterior cortical areas, reflecting alterations of visual processing by motivational properties.

\section{Method}

\section{Participants}

Eleven right-handed, male students with normal or corrected-tonormal vision whose age ranged from 24 to 30 years (mean age 26 years) gave informed consent to participate in the study. They either were given class credits or paid $10 \mathrm{DM}$.

\section{Stimuli and Design}

Sixty colored pictures were selected from the IAPS (Center for the Study of Emotion and Attention, 1999) consisting of 20 pleasant, 20 neutral, and 20 unpleasant pictures, based on pleasure and arousal ratings. Pleasant pictures included erotic couples and happy families; neutral pictures included neutral faces and household objects; unpleasant pictures included mutilated bodies and scenes of attack and threat. ${ }^{1}$ Pleasant and unpleasant pictures were se-

${ }^{1}$ The numbers of IAPS pictures used were as follows. Pleasant: 2050 , 2070, 2080, 2160, 2165, 2170, 2311, 2340, 2341, 2360, 4650, 4651, 4652, 4658, 4659, 4660, 4664, 4670, 4680, 4690. Neutral: 2190, 2200, 2210, 2230, 2381, 2440, 2480, 2570, 2850, 7002, 7009, 7010, 7020, 7030, 7040, 7080, 7175, 7233, 7235, 9070. Unpleasant: 1050, 1120, 1201, 1300, 1930, $3000,3010,3050,3060,3071,3080,3102,3110,3130,3530,6260,6350$, $6510,6540,9405$. lected to be the most arousing exemplars in the respective subcategory. Normative ratings of valence for pictures in these categories differed (pleasant: 7.4, neutral: 4.9, unpleasant: 2.6), as did normative ratings of arousal (pleasant: 5.7, neutral: 2.6; unpleasant, 6.4; Lang, Bradley, \& Cuthbert, 1999). Each picture was presented on a 19-in. computer screen with a frame refresh rate of $60 \mathrm{~Hz}$. The screen was placed $1.5 \mathrm{~m}$ in front of the viewer, resulting in a picture presentation with a visual angle of $15^{\circ}$ horizontally and $11^{\circ}$ vertically. Each picture was presented for $6,000 \mathrm{~ms}$, with intertrial intervals varying between 7,000 and 12,000 ms.

\section{Electrophysiological Recordings}

EEG was recorded continuously from 129 electrodes using an Electrical Geodesics ${ }^{\mathrm{TM}}$ (EGI) high-density EEG system and digitized at a rate of $250 \mathrm{~Hz}$, using $\mathrm{Cz}$ as a recording reference. Impedances were kept below $50 \mathrm{k} \Omega$, as recommended for the Electrical Geodesics high input-impedance amplifiers. A subset of EGI net electrodes located at the outer canthi as well as above and below the right eye was used to determine horizontal and vertical electrooculogram (EOG). All channels were preprocessed on-line by means of $0.1-\mathrm{Hz}$ high-pass and $100-\mathrm{Hz}$ low-pass filtering.

\section{Procedure}

In a first training procedure, participants were presented with two exemplars from each affective category. These pictures were not part of the experimental set. During this procedure, participants were trained to maintain gaze on the center of the screen and to avoid explorative eye movements. To this end, the experimenter, who visually inspected the participants' eye movements in response to stimuli, provided direct feedback. Subsequently, the sensor net was applied and participants viewed two blocks, each consisting of the same 60 pictures. The order of the stimuli was pseudo-randomized with the restriction that no more than three pictures in the same affective category could occur in a row. Subjects were instructed to avoid eye movements and eye blinks and view the pictures while they were on the screen.

After the EEG recordings, participants viewed the 60 pictures again in a pseudo-randomized order and were asked to rate each picture for pleasure and arousal using the paper and pencil version of the Self-Assessment Manikin (SAM; Lang, 1980). SAM is a language-free instrument for rating pleasure and arousal, and consists of a graphic figure representing nine levels each of pleasure and arousal. SAM ratings of affect correlate highly with longer, verbally oriented instruments such as the semantic differential scale (Bradley \& Lang, 1994).

\section{Data Reduction and Analysis}

Epochs of 6,200 ms (200 ms pre-, 6,000 ms postonset) were obtained for each stimulus from the continuously recorded EEG. The mean voltage of a 200 -ms segment preceding picture onset was subtracted as the baseline. In a first step, data were low-pass filtered at a frequency of $40 \mathrm{~Hz}$ ( $24 \mathrm{~dB} /$ octave) and then submitted to the procedure proposed by Junghöfer, Elbert, Tucker, and Rockstroh (2000). This procedure uses statistical parameters of the data to exclude channels and trials that are contaminated with artifacts. Recording artifacts are first detected using the recording reference (i.e., $\mathrm{Cz}$ ), and then global artifacts are detected using the average reference. In a next interactive step, distinct sensors from particular trials are removed based on the distribution of their amplitude, standard deviation, and gradient. Data at eliminated electrodes are replaced with a statistically weighted spherical spline interpolation from the full channel set (Junghöfer, Elbert, Leiderer, Berg, \& 
Rockstroh, 1997). In a last step, the variance of the signal across trials is computed to determine the stability of the average waveform.

The mean number of approximated channels across conditions and subjects was 14 . With respect to the spatial arrangement of the approximated sensors, it was ensured that the rejected sensors were not located within one region of the scalp, as this would make interpolation for this area invalid. Spherical spline interpolation was used throughout both for approximation of sensors and illustration of voltage maps (Junghöfer et al., 1997; Perrin, Pernier, Bertrand, Giard, \& Echallier, 1987). Single epochs with excessive eye movements and blinks or more than 20 channels containing artifacts were discarded. The validity of this procedure was further tested by visually inspecting the vertical and horizontal EOG as computed from a subset of the electrodes that were part of the electrode net. Trials that showed remaining ocular artifacts were dismissed at this step of the analysis. Subsequently, data were arithmetically transformed to the average reference, which was used for all analyses. After artifact correction, an average of $69 \%$ of the 120 trials was retained in the analyses, with a mean of 28.6 (range: 22 to 37 ) trials for the neutral pictures, 26.4 (range: 23 to 34 ) for the pleasant, and 27.2 (range: 21 to 36 ) for the unpleasant pictures.

As a measure of global cortical activity and assessment of ERP peaks and their respective time course, we computed the Global Power $g(t)$ of the ERP (Skrandies, 1995). This metric was computed as the mean square of the averaged signal across electrodes for each emotional content, weighted by the standard deviation of the voltage across trials (Junghöfer et al., 2000), at each electrode and time point (see Equation 1).

$$
g(t)=\frac{\sum_{j=1}^{128} x_{j}(2)^{2} \cdot s_{j}(t)^{-1}}{128 \cdot \sum_{j=1}^{128} s_{j}(t)^{-1}},
$$

where $x_{j}(t)$ is the voltage at sensor $j$ and time $t$ and $s_{j}(t)$ is the standard deviation of the voltage at sensor $j$ and time $t$ across trials.

Cortical sources were estimated using a minimum norm estimate, a linear estimation technique first proposed by Hämäläinen and Ilmoniemi (1984). Here we used the implementation suggested by Hauk and collaborators (2002). Code written in MATLAB ${ }^{\mathrm{TM}}$ was applied to the voltage data. The minimum norm estimate is an inverse method for reconstructing the primary current that underlies an extracranially recorded time-locked brain potential. The origin of the signal is estimated using multiple model dipoles, each being equidistantly arranged on three concentric shells. The procedure is based on the assumption that the data vector $\mathbf{d}$, which contains the recorded scalp potential at given electrode sites, can be described as the product of the leadfield matrix $\mathbf{L}$, which specifies the electrode's sensitivity to the sources, the source current vector $\mathbf{j}$ and a noise component $\epsilon$ (Grave de Peralta Menendez, Hauk, Gonzalez Andino, Vogt, \& Michel, 1997).

Since $\mathbf{L}$ and $\mathbf{d}$ are known, and $\epsilon$ is treated as if to be estimated with an acceptable accuracy, the minimum norm estimate for $\mathbf{j}$ is the mathematically unique solution of the equation that minimizes the squared current density $\left(\mathbf{j}^{2}=\min \right)$. This solution is obtained by multiplying the pseudoinverse of the leadfield matrix $\mathbf{L}$ with the data. Given the high number of electrodes and the presence of noise, spatial regularization is performed with the factor $\lambda$. To this end, Tikhonov-Phillips regularization for matrices was applied during pseudo-inversion of the leadfield matrix $\mathbf{L}$ (i.e., $\mathbf{L}+\lambda \mathbf{I}$ is pseudo-inverted, with I being the identity matrix; Hauk et al., 2002). In the present work, the regularization $\lambda=.01$ was used throughout. An important feature of this algorithm is that sources that do not contribute to the measured scalp potential are omitted. A priori information about the number or locations of cortical sources is not required.

One issue concerning the accuracy of inverse solutions is the role of the depth of the sources. The present algorithm addresses this problem in the following manner: A three-dimensional source space consisting of three concentric shells $(80 \%, 60 \%$, and $40 \%$ of electrode radius) is computed as a rough approximation of the brain volume. Subsequently, an inverse solution is determined for these shells. Estimates of the minimum norm method for deep sources are not independent from superficial ones however, which is due to the general constraints imposed by the inverse problem of bioelectric processes (Ilmoniemi, 1993). The solutions for the different shells can be considered as indices of the true current source differing with respect to their degree of blurring and depth sensitivity. Accordingly, it has been shown for the biomagnetic case that "deeper" shells are associated with less suppression of deep sources, but more blurring occurs (Hauk, Berg, Wienbruch, Rockstroh, \& Elbert, 1998). A significant difference between shells is, thus, that the most superficial shell has the best spatial resolution, and the deepest shell has the worst, whereas the opposite is true for depth sensitivity. For this reason, we report the solutions for the shell at $60 \%$ electrode radius throughout. Simulations have shown that this shell yields the best signal-to-noise ratio for simulated dipolar sources across different depth and amplitude parameters (Hauk et al., 2002). The 129 locations on this shell were selected to correspond to the recording sites of the EGI system, and the respective minimum norm estimate amplitudes at these sites were computed for further analysis.

\section{Statistical Analysis}

For the purpose of statistical analysis, both the ERP voltages at 129 electrodes and the dipole strengths provided by the minimum norm estimates at each of the respective 129 sites were averaged for 12 regions, formed by crossing hemisphere (left, right) with horizontal plane (anterior, lateral, posterior), and vertical plane (inferior, superior), based on recording sites of the international 10-20 system. Mean voltages and mean dipole strength in these regions were assessed in five time windows, corresponding roughly to P1 (70$90 \mathrm{~ms}), \mathrm{N} 1$ (120-150 ms), an early P3 window (300-340 ms), a later P3 window (380-440 ms), and a slow wave window (550$900 \mathrm{~ms}$ ). These data were submitted to repeated-measures analysis of variance (ANOVA) with factors of picture content (3 levels: pleasant, neutral, unpleasant), hemisphere (2 levels: left, right), and region (6 levels: antero-inferior, antero-superior, lateralinferior, lateral-superior, postero-inferior, postero-superior). The locations of these regions with respect to sites of the international 10-20 system are shown in Figure 1. Time windows were selected on the basis of the grand mean global power for those segments that contained the most pronounced voltage peaks.

This has the additional advantage that minimum norm source space projection is done with data showing a satisfactory signalto-noise ratio. For assessment of interactions between emotional content and topographical factors (i.e., region and hemisphere) ERP and dipole strength data were normalized as proposed by McCarthy and Wood (1985; see also Lykken, 1972). For each condition and time window, the minimum and maximum values across electrodes were determined and the normalized value $n$ at each electrode $j$ was computed according to 


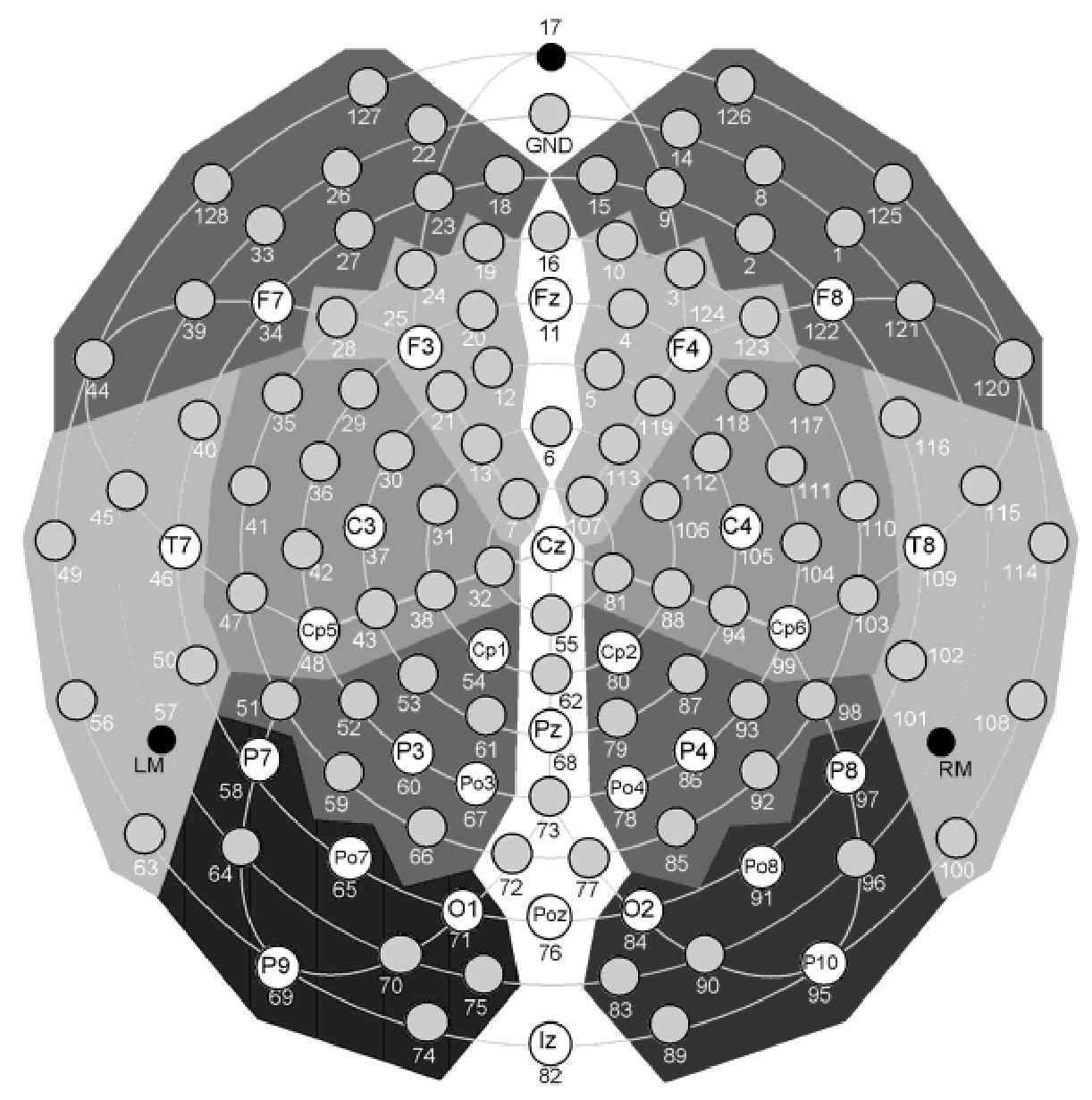

Figure 1. Layout of the electrode array. Electrodes in the shaded clusters were grouped for statistical analysis. Frontal electrodes are shown at the top of the figure. RM and LM denote right and left mastoids, respectively.

$$
n_{j}(t)=\frac{x_{j}(t)-\min }{\max -\min }
$$

where $x_{j}(t)=$ potential at sensor $j$ and time $t$.

For evaluation of significant $(p<.05)$ main effects and interactions, critical differences were determined using the Scheffé procedure. The SAM pleasure and arousal ratings were evaluated by means of one-way ANOVAs with a factor of picture content (pleasant, neutral, and unpleasant). Where appropriate, degrees of freedom were adjusted using the Greenhouse-Geisser method (Greenhouse \& Geisser, 1959). In the following, uncorrected $F$ values are reported together with the Greenhouse-Geisser epsilon and corrected $p$ values.

\section{Results}

\section{SAM Ratings}

As expected, SAM pleasure ratings differed as a function of picture content, $F(2,20)=259.94, p<.001, \epsilon=.87$, with pleasant (mean pleasure rating: 7.1), neutral (4.9), and unpleasant pictures (2.2) all rated as significantly different from each other. Similarly, arousal ratings also differentiated picture categories, $F(2,20)=$ 169.69, $p<.001, \epsilon=.91$, with higher arousal ratings for both pleasant pictures (Scheffé $p<.01$, mean arousal rating: 5.2) and unpleasant pictures (Scheffé $p<.01$ mean arousal rating: 6.4) than for neutral pictures (mean arousal rating: 2.2). Unpleasant pictures were also rated as slightly more arousing than pleasant pictures, $p<.01$, which was consistent with the IAPS normative ratings.

\section{ERP Scalp Voltages}

ERP traces at electrodes corresponding to 30 sites of the international 10-20 system are shown in Figure 2. The squared electrical activity over all sensors, trials, and subjects, that is, the global power plot, is shown in Figure 3. Given our hypothesis of differential involvement of visual cortex for different emotional contents, the focus of the present study was on effects at posterior sites.

Positivity in the P1 (70-90 ms) time window was greatest over the posterior sites, both inferior and superior, main effect of region, $F(5,50)=6.4, p<.01, \epsilon=.51$. No significant main effects or interactions involving picture content were found in this time segment.

Mean voltage in the N1 (120-150 ms) time window resulted in a Content $\times$ Region interaction, $F(10,100)=3.8, p<.05, \epsilon=.30$. Table 1 lists these means. Scheffé tests indicated that content effects were present at anterior, lateral-inferior, and posteroinferior sites. In particular, antero-superior and postero-inferior sites showed enhanced amplitude for pleasant pictures, compared to neutral and unpleasant pictures, $p<.05$.

Pronounced main effects of picture content were also seen in both the early P3 (300-400 ms), $F(2,20)=12.1, p<.01, \epsilon=.94$, and the late P3 (380-440 ms) time windows, $F(2,20)=7.4, p<$ $.01, \epsilon=.68$, with greater voltage in each temporal region when 


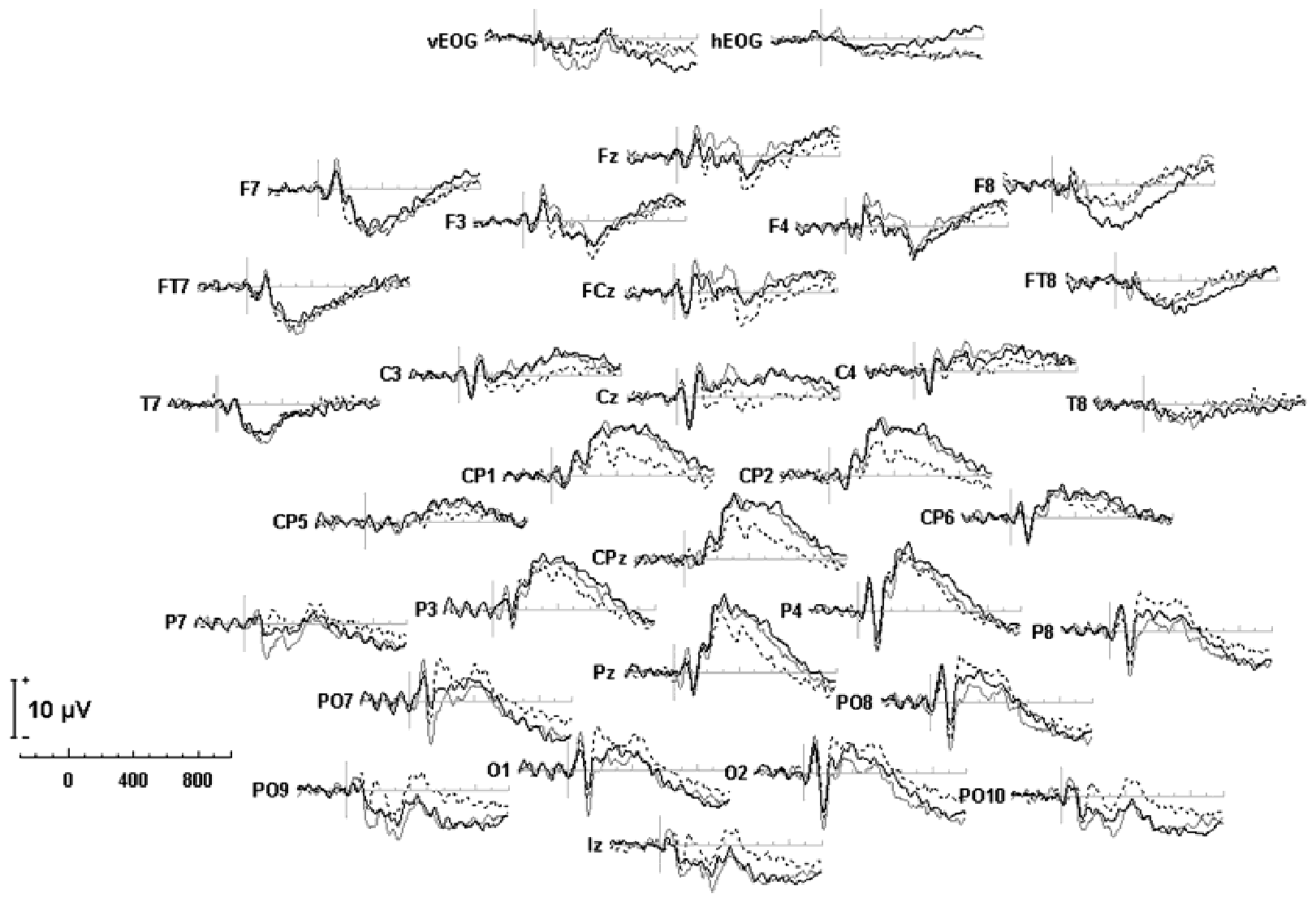

Figure 2. Grand mean $(N=11)$ event-related potentials at selected electrodes corresponding to sites of the International 10-20 System, for pleasant (gray), neutral (dashed), and unpleasant (black) content. Note: Positive is up.

viewing either pleasant or unpleasant pictures compared to neutral pictures, $p<.05$. A main effect of region showed that posterosuperior sites contributed most to the positive potential in both the early $\mathrm{P} 3, F(5,50)=48.2, p<.01, \epsilon=.54$, and the late $\mathrm{P} 3$ temporal regions, $F(5,50)=25.8, p<.01, \epsilon=.38$. Furthermore, Content $\times$ Region interactions were obtained for both the early P3, $F(10,100)=3.2, p<.05, \epsilon=.25$, and for the late $\mathrm{P} 3$ windows, $F(10,100)=7.6, p<.01, \epsilon=.27$, which indicated that these effects differed across regions. In the early P3 window, anterosuperior and postero-inferior sites showed a voltage amplitude enhancement for pleasant, compared to neutral and unpleasant content, whereas lateral-inferior and postero-superior sites showed discrimination between emotionally arousing and neutral pictures, $p<.05$. The latter effects were most pronounced at righthemispheric regions (see Table 1).

Interestingly, activity in the late $\mathrm{P} 3$ window differed somewhat from the early P3 in that differences related to picture content were lateralized for the late P3 temporal region. The three way Content $\times$ Hemisphere $\times$ Region interaction was significant for late P3 temporal windows, $F(10,100)=6.1, p<.01, \epsilon=.19$, and showed that positivity at lateral-superior and postero-superior regions was enhanced for emotional compared to neutral content at left-

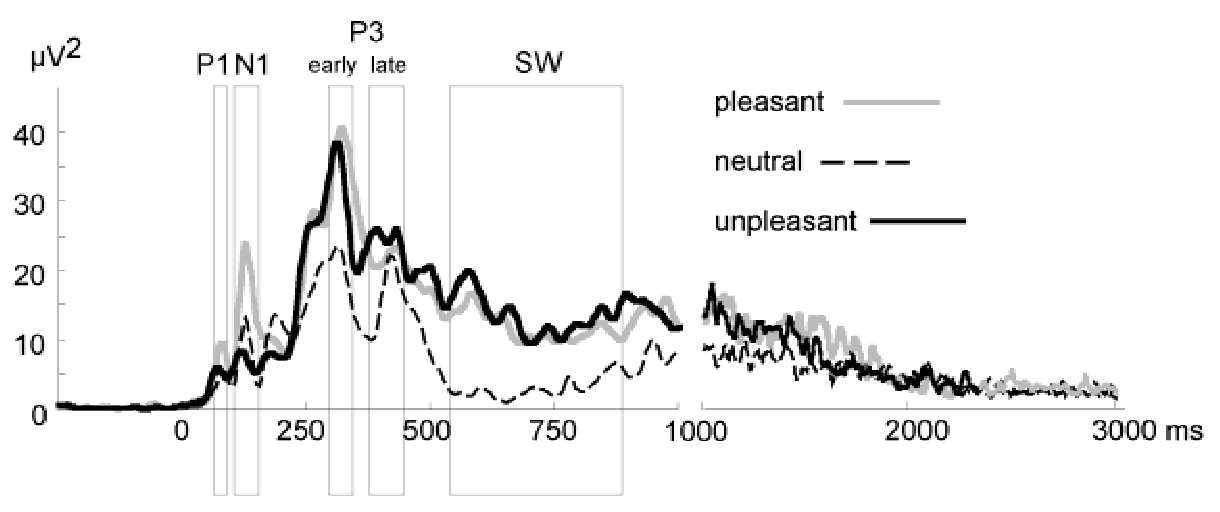

Figure 3. Grand mean $(N=11)$ global power (see Equation 1) of the voltage obtained at 129 scalp sites for pleasant, neutral, and unpleasant picture content. 
Table 1. Mean Voltages ( \pm SEM) in the N1, Early P3, Late P3 and Slow Wave Windows, at Six Regions in the Left and Right Hemisphere (See Figure 1), for Pleasant, Neutral, and Unpleasant Picture Content

\begin{tabular}{|c|c|c|c|c|c|c|}
\hline \multirow[b]{2}{*}{ Window } & \multicolumn{3}{|c|}{ Left hemisphere } & \multicolumn{3}{|c|}{ Right hemisphere } \\
\hline & Pleasant & Neutral & Unpleasant & Pleasant & Neutral & Unpleasant \\
\hline \multicolumn{7}{|l|}{ N1 $(120-150 \mathrm{~ms})$} \\
\hline Antero-inferior & $0.51 \pm 0.46$ & $-0.16 \pm 0.39$ & $0.34 \pm 0.42$ & $0.71 \pm 0.66$ & $-0.12 \pm 0.56$ & $-0.92 \pm 0.70$ \\
\hline Antero-superior & $1.82 \pm 0.45$ & $0.71 \pm 0.44$ & $0.62 \pm 0.47$ & $2.16 \pm 0.56$ & $1.06 \pm 0.57$ & $0.76 \pm 0.61$ \\
\hline Lateral-inferior & $-1.86 \pm 0.56$ & $-0.95 \pm 0.23$ & $-1.18 \pm 0.42$ & $-1.71 \pm 0.47$ & $-1.10 \pm 0.26$ & $-1.30 \pm 0.48$ \\
\hline Lateral-superior & $0.94 \pm 0.40$ & $0.78 \pm 0.46$ & $0.73 \pm 0.39$ & $1.28 \pm 0.48$ & $0.86 \pm 0.66$ & $0.71 \pm 0.45$ \\
\hline Postero-inferior & $-2.68 \pm 0.77$ & $-0.82 \pm 0.54$ & $-0.81 \pm 0.62$ & $-2.58 \pm 0.69$ & $-1.61 \pm 0.32$ & $-1.02 \pm 0.80$ \\
\hline Postero-superior & $-0.16 \pm 0.60$ & $0.29 \pm 0.42$ & $0.82 \pm 0.27$ & $-0.73 \pm 0.62$ & $-0.63 \pm 0.54$ & $0.24 \pm 0.54$ \\
\hline \multicolumn{7}{|c|}{ Early P3 (300-400 ms) } \\
\hline Antero-inferior & $-3.24 \pm 0.78$ & $-2.82 \pm 0.69$ & $-2.99 \pm 0.68$ & $-2.13 \pm 0.73$ & $-1.74 \pm 0.41$ & $-2.39 \pm 0.59$ \\
\hline Antero-superior & $0.37 \pm 0.31$ & $-0.61 \pm 0.56$ & $-0.67 \pm 0.48$ & $1.07 \pm 0.36$ & $-0.18 \pm 0.39$ & $-0.25 \pm 0.42$ \\
\hline Lateral-inferior & $-4.07 \pm 0.49$ & $-2.92 \pm 0.46$ & $-3.21 \pm 0.62$ & $-2.83 \pm 0.46$ & $-1.55 \pm 0.43$ & $-2.36 \pm 0.54$ \\
\hline Lateral-superior & $0.21 \pm 0.32$ & $-0.79 \pm 0.21$ & $-0.09 \pm 0.31$ & $1.51 \pm 0.39$ & $0.91 \pm 0.36$ & $0.90 \pm 0.42$ \\
\hline Postero-inferior & $-1.76 \pm 0.39$ & $-0.28 \pm 0.52$ & $-0.57 \pm 0.56$ & $-0.98 \pm 0.37$ & $0.63 \pm 0.50$ & $0.02 \pm 0.44$ \\
\hline Postero-superior & $2.89 \pm 0.59$ & $2.53 \pm 0.60$ & $3.48 \pm 0.40$ & $3.99 \pm 0.55$ & $3.13 \pm 0.48$ & $4.30 \pm 0.45$ \\
\hline \multicolumn{7}{|c|}{ Late P3 $(380-440 \mathrm{~ms})$} \\
\hline Antero-inferior & $-3.13 \pm 0.69$ & $-2.80 \pm 0.70$ & $-2.45 \pm 0.77$ & $-2.07 \pm 0.59$ & $-1.29 \pm 0.53$ & $-3.56 \pm 0.77$ \\
\hline Antero-superior & $-1.51 \pm 0.60$ & $-2.71 \pm 0.46$ & $-1.59 \pm 0.59$ & $-1.31 \pm 0.55$ & $-2.45 \pm 0.52$ & $-1.98 \pm 0.59$ \\
\hline Lateral-inferior & $-1.70 \pm 0.36$ & $-0.76 \pm 0.36$ & $-1.11 \pm 0.44$ & $-1.03 \pm 0.38$ & $0.40 \pm 0.31$ & $-1.38 \pm 0.44$ \\
\hline Lateral-superior & $0.41 \pm 0.43$ & $-0.87 \pm 0.33$ & $0.43 \pm 0.43$ & $0.85 \pm 0.32$ & $-0.02 \pm 0.41$ & $0.19 \pm 0.43$ \\
\hline Postero-inferior & $0.09 \pm 0.58$ & $1.76 \pm 0.57$ & $0.61 \pm 0.61$ & $0.02 \pm 0.59$ & $1.97 \pm 0.54$ & $0.56 \pm 0.61$ \\
\hline Postero-superior & $3.22 \pm 0.52$ & $2.65 \pm 0.49$ & $3.58 \pm 0.40$ & $3.09 \pm 0.44$ & $2.89 \pm 0.39$ & $3.43 \pm 0.40$ \\
\hline \multicolumn{7}{|c|}{ Slow wave $(550-900 \mathrm{~ms})$} \\
\hline Antero-inferior & $-0.89 \pm 0.51$ & $-0.56 \pm 0.42$ & $-0.42 \pm 0.51$ & $0.17 \pm 0.68$ & $0.68 \pm 0.47$ & $-1.32 \pm 0.59$ \\
\hline Antero-superior & $0.82 \pm 0.35$ & $-0.13 \pm 0.33$ & $0.56 \pm 0.44$ & $0.85 \pm 0.52$ & $-0.16 \pm 0.42$ & $0.11 \pm 0.49$ \\
\hline Lateral-inferior & $-1.45 \pm 0.38$ & $-0.41 \pm 0.26$ & $-1.29 \pm 0.42$ & $-1.34 \pm 0.32$ & $0.01 \pm 0.27$ & $-1.79 \pm 0.33$ \\
\hline Lateral-superior & $1.42 \pm 0.28$ & $0.43 \pm 0.18$ & $1.46 \pm 0.26$ & $1.46 \pm 0.22$ & $0.69 \pm 0.21$ & $1.11 \pm 0.23$ \\
\hline Postero-inferior & $-1.87 \pm 0.46$ & $-0.33 \pm 0.40$ & $-1.53 \pm 0.50$ & $-2.41 \pm 0.51$ & $-0.76 \pm 0.39$ & $-1.84 \pm 0.42$ \\
\hline Postero-superior & $1.27 \pm 0.35$ & $0.63 \pm 0.31$ & $1.81 \pm 0.36$ & $0.65 \pm 0.29$ & $0.33 \pm 0.28$ & $1.41 \pm 0.31$ \\
\hline
\end{tabular}

hemispheric sites, $p<.05$ (see Table 1). On the right hemisphere, there was a different pattern, with enhanced positivity for pleasant content at lateral-superior sites, and unpleasant content being associated with enhanced positivity at lateral-superior sites, $p<.05$. Anterior-inferior and lateral inferior sites on the right hemisphere showed the same pattern with higher negative amplitudes for emotionally arousing pictures, $p<.01$. Across hemispheres, anterosuperior sites showed greater negativity for neutral compared to emotional content, $p<.05$.

The slow wave window (550-900 ms) exhibited a main effect of picture content, $F(2,20)=13.3, p<.01, \epsilon=.75$, reflecting greater ERP amplitudes for pleasant and unpleasant as compared to neutral content, $p<.01$. A significant interaction of Content $\times$ Hemisphere $\times$ Region, $F(10,100)=3.7, p<.05, \epsilon=.31$, showed that over the left hemisphere, these content effects were significant at superior regions and at the postero-inferior regions, $p<.05$ (see Table 1). Over the right hemisphere, significantly enhanced amplitudes for affectively arousing pictures were seen at all inferior regions and the antero-superior region, $p<.05$. In addition, positivity for unpleasant pictures was significantly greater than neutral or pleasant pictures at the right postero-superior region, $p<.05$. Overall amplitudes were smaller on the left hemisphere, but there was a significant enhancement for arousing compared to neutral pictures at the two posterior regions as well as at the lateral superior region, $p<.05$.

\section{Minimum Norm Estimate}

To assess effects of picture content on estimated source parameters of electrocortical activity, we conducted the same statistical analy- ses as reported above for the dipole strengths estimated using the minimum norm procedure. Paralleling the voltage data, no significant main effects or interactions involving picture content were found in the P1 window.

In contrast, dipole strength was affected by picture content in the N1 time window (Figure 4, left), $F(2,20)=10.11, p<.01, \epsilon=$ .87 , illustrated in Figure 5, top panel. Scheffé tests indicated enhanced dipole strength when viewing pleasant pictures, compared to either neutral, $p<.05$, or unpleasant, $p<.05$, pictures. No interaction involving region or hemisphere was found, indicating that contents differed with respect to source strength rather than changes in source distribution.

In the early P3 time window, a main effect of picture content, $F(2,20)=6.4, p<.05, \epsilon=.88$, indicated that pleasant and unpleasant pictures were generally associated with greater dipole strength than were neutral pictures, $p<.05$. A Content $\times$ Hemisphere interaction (Figure 5), $F(2,20)=4.7, p<.05, \epsilon=.98$, showed that effects of picture content differed across hemispheres. Follow-up tests demonstrated that right-hemisphere sources discriminated between emotional (pleasant or unpleasant) and neutral pictures, whereas the left hemisphere showed a significant enhancement for pleasant stimuli only, compared to neutral pictures, $p<.05$ (Figure 4, middle).

In the late $\mathrm{P} 3$ window, the Content $\times$ Hemisphere interaction, $F(2,20)=4.1, p<.05, \epsilon=.94$, indicated that effects were restricted to the right hemisphere (Figure 5), with unpleasant picture processing associated with greater dipole activity than neutral pictures, $p<.05$, whereas other differences did not reach significance. 
N1 (120-150 ms)

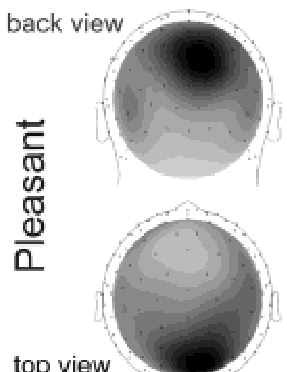

(1)
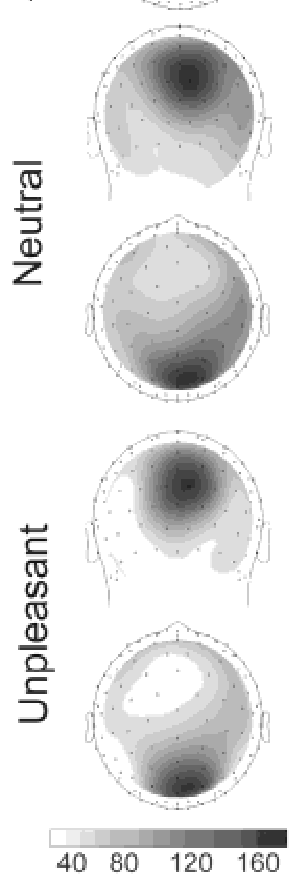

early $\mathrm{P} 3$ (300-340 ms)
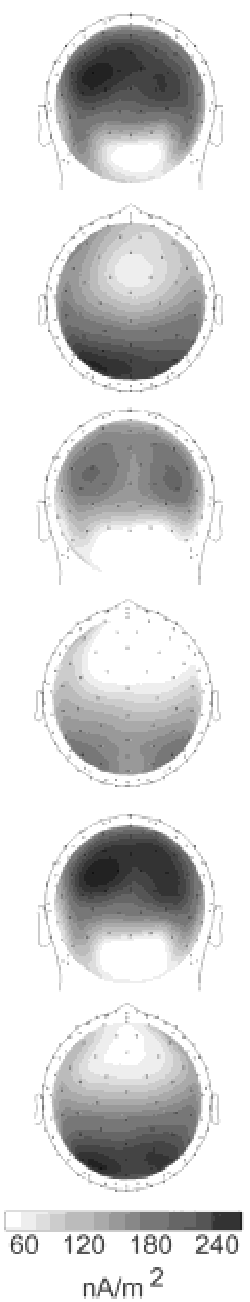

SW
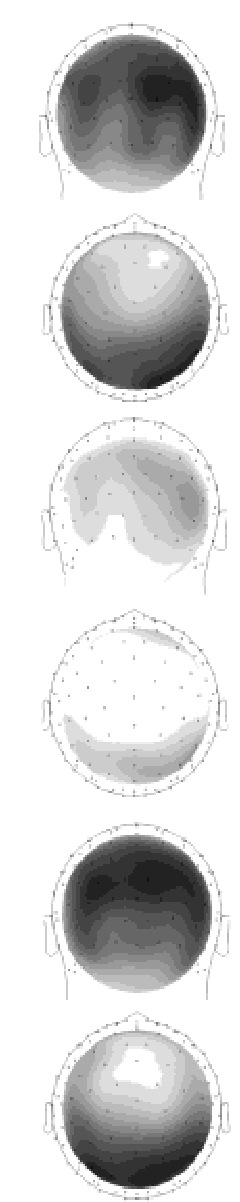

$\begin{array}{llll}60 & 80 & 100 & 120\end{array}$
(550-900 ms)

Figure 4. Grand mean $(N=11)$ topography of MNE dipole strength for the N1, early P3, and slow wave (SW) deflection of the ERP for pleasant, neutral, and unpleasant picture content. Note: Scaling is different for each time window.

A main effect of picture content was obtained for the slow wave window (550-900 ms), $F(2,20)=778, p<.01, \epsilon=.98$ (see Figure 4, right). This effect was due to an enhancement of dipole strength when viewing emotionally arousing compared to neutral pictures, $p<.01$. These differences were significant at posterosuperior, posterior-inferior, lateral-inferior, and lateral-superior sites, Content $\times$ Region, $F(10,100)=2.9, p<.05, \epsilon=.32$, whereas anterior sites showed the opposite pattern, with neutral stimuli showing enhanced dipole strength compared to viewing emotional pictures, $p<.05$, which did not differ from each other (see Figure 5, bottom).

\section{Discussion}

The present study aimed at replicating and extending previous ERP work with affective pictures, using a dense-array EEG montage and a distributed source modeling technique. As found in previous studies, differences in the magnitude of positive-going
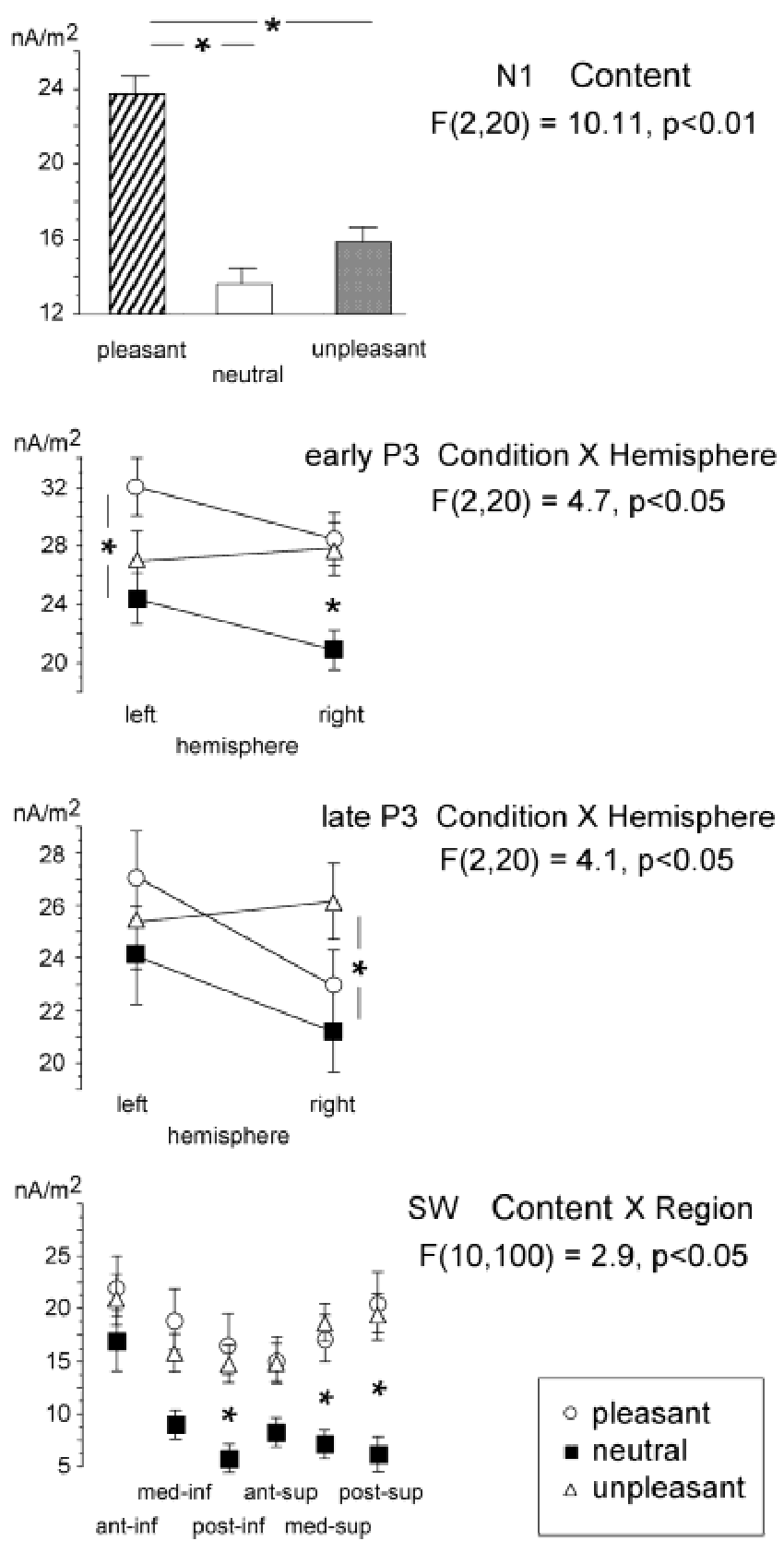

Figure 5. Graphical representation of effects of picture content on minimum norm dipole strength for the N1, early P3, late P3, and SW time windows. Error bars represent standard errors.

potentials in time windows later than $300 \mathrm{~ms}$ were found as a function of emotional arousal (e.g., Cuthbert et al., 2000). This effect was largest at electrode sites near $\mathrm{Pz}$ and showed a broad distribution, both in the voltage domain and in source space projection using the minimum norm estimate. In this respect, the polarity and topography of the effect are similar to that seen in previous work, which is surprising, given the increase in montage size and the use of an average rather than linked mastoid reference. On the other hand, using a higher number of electrodes, MNE, and the average reference, effects of emotional content were strongest in posterior sites, near occipital-temporal cortex and posterior parietal cortex. Thus, the results obtained with the present method 
seem topographically specific, whereas modulations by emotional content were more equivalent across the entire scalp using sparse electrode arrays. Further, as could be expected from the neuroanatomy of human visual cortex, modulation by arousal was most pronounced at locations off the midline, providing more data on activity in the visual system than has been available with sparse electrode arrays. Regional MNE effects of emotional content at posterior sites supported this view. Thus, the cortical networks for sensory processing may be subject to gain mechanisms according to the motivational relevance of the visual stimuli, as has been proposed for attentional mechanisms (Hillyard \& Anllo-Vento, 1998).

Examining the differences between affective contents in terms of scalp voltage in six time windows, we found no stable hemispheric asymmetry but rather changing preponderance over the course of time as well as anterior-posterior differences regarding lateralization. Findings obtained with average reference and MNE converged regarding these effects. The earliest time windows (P1, N1) showed no differential hemispheric activity, whereas late P3 and slow wave displayed more reactivity to emotionally arousing (pleasant or unpleasant) pictures over the right hemisphere, as has been reported earlier for comparable time ranges (Crites, Cacioppo, Gardner, \& Berntson, 1995). The late P3 showed specific enhancement for pleasant pictures over left-hemisphere regions. The slow wave was sensitive to emotional arousal over different regions in the left and right hemispheres.

However, as illustrated in the topographical maps of estimated dipole strength (Figure 4), asymmetries were also present for the neutral pictures, and, in general, content effects were most likely in regions with high signal. Thus, right-hemispheric dominance in emotional picture viewing may be partly due to picture perception per se, rather than a specific concomitant of emotional processing. Alternatively, changes of motivational state and mood during viewing affective pictures in the experimental session might affect processing of the neutral pictures. Presenting neutral pictures of the IAPS alone may test these speculations. As suggested by classic visual ERP work with simple pattern stimuli (e.g., Shagass, 1972; Shagass, Amadeo, \& Roemer, 1976), temporally changing patterns of ERP hemisphere differences may reflect hemispheric interactions that are part of visual picture processing rather than effects of hemispheric specialization for different emotions. It seems likely that in the present paradigm, motivated attention is associated with changes in response amplitude in active tissue, showing only moderate changes in hemispheric asymmetries, rather than exclusive involvement of a specific hemisphere. These modest changes point towards a stronger involvement of the right hemisphere, which is consistent with neuropsychological (Borod et al., 1998) and psychophysiological data (Kayser et al., 1997; Keil et al., 2001).

An enhancement of voltage and dipole strength in the N1 temporal window was found for pleasant pictures. The source areas of this effect were located in occipital visual cortices. It is unclear whether this reflects differences in physical stimulus parameters or results from a selective perceptual mechanism. In a visual hemifield design employing the same set of IAPS pictures, Keil et al. (2001) found an N1 enhancement for arousing, compared to neutral pictures. Likewise, early ERP differences between arousing and neutral stimuli have been found when pictures are presented very rapidly (Junghöfer, Bradley, Elbert, \& Lang, 2001). In the current study, this would suggest that effects due to emotional arousal should be greater for unpleasant pictures, which were rated as slightly more arousing than pleasant pictures. We did, however, observe the opposite pattern, with pleasant pictures showing the most pronounced N1 enhancement. Given that the present sample contained only males, replication of the findings regarding not only the $\mathrm{N} 1$, but also regarding later deflections is desirable. Generally, gender effects have repeatedly been observed with psychophysiological measures and affective picture stimuli (Lang et al., 1993). For instance, using fMRI, Lang et al. (1998) have found gender differences showing more pronounced visual cortex activation during presentation of pleasant material in men, compared to female participants. For the present study, this suggests that the specific voltage enhancement for pleasant content as found in the $\mathrm{N} 1$ as well as in several regions in both P3 components may not be seen in a female sample. Thus, replication in a larger sample with the longer presentation period used here is necessary before one draws strong conclusions regarding the modulation of early components by affect.

One aim of the present study was to use the minimum norm estimation procedure to complement the findings obtained by assessing the voltage data using a dense electrode array. We found convergent results regarding effects of emotional content for both the average referenced voltages and the MNE dipole strength values. As illustrated in the topographical maps of dipole strength for the early P3 and the slow wave components (Figure 4), maxima in dipole strength were seen in occipital regions, while at the same time the voltage maps showed increasing gradients between posteroinferior and postero-superior sensors. This is consistent with previous findings using distributed source modeling that found that generators tangentially oriented to the surface of the scalp are associated with broad voltage distributions (Pascual-Marqui et al., 1994). These distributions might in turn be misinterpreted as being due to one or more source structures that are oriented radially. It is striking, however, that the results obtained using distributed modeling converged with those obtained using the average referenced voltage data, both finding modulation of late components as a function of emotional arousal primarily at posterior areas.

In addition, examining the spatio-temporal dynamics of the event-related potential in the source space revealed that, with increasing viewing time, the regions showing affect modulation extended from inferior-posterior to higher order visual cortical areas such as parietal and occipito-temporal regions. This finding is consistent with other ERP studies of visual information processing (Luck \& Hillyard, 1995) as well as studies of induced oscillatory brain activity in affective picture perception (Müller, Keil, Gruber, \& Elbert, 1999). For instance, whereas the dipole strength map in the P3 temporal windows showed its maximum at posterior and parietal sites, the late positive slow wave showed additional activity originating at lateral-inferior and anterior regions.

Most interestingly, the topographical distribution of effects for dipole strength in the slow wave regions showed remarkable similarity to the findings obtained using fMRI during affective picture processing (Lang et al., 1998), with the greatest activity for emotional pictures occurring in posterior and lateral sites associated with occipito-temporal cortex. This suggests that signals may primarily reflect slow wave EEG activity, that is, information processing that is sustained. Slow wave activity occurred in a time window of several seconds and probably is a correlate of longer lasting, higher order processing in occipito-temporal and temporal structures. Modulation of the cortical slow wave, as well as the blood-oxygen-level-dependent response, by emotional arousal may reflect a relatively high level of visual processing, in which sustained attention is allocated to motivationally relevant and emotionally salient cues. 


\section{REFERENCES}

Borod, J. C., Cicero, B. A., Obler, L. K., Welkowitz, J., Erhan, H. M., Santschi, C., Grunwald, I. S., Agosti, R. M., \& Whalen, J. R. (1998). Right hemisphere emotional perception: Evidence across multiple channels. Neuropsychology, 12, 446-458.

Bradley, M. M., Greenwald, M. K., Petry, M. C., \& Lang, P. J. (1992). Remembering pictures: Pleasure and arousal in memory. Journal of Experimental Psychology: Learning, Memory, \& Cognition, 18, 379-390.

Bradley, M. M., \& Lang, P. J. (1994). Measuring emotion: The SelfAssessment Manikin and the semantic differential. Journal of Behavior Therapy \& Experimental Psychiatry, 25, 49-59.

Bradley, M. M., Lang, P. J., \& Cuthbert, B. N. (1993). Emotion, novelty, and the startle reflex: Habituation in humans. Behavioral Neuroscience, 107, 970-980.

Cacioppo, J. T., Crites, S. L., Jr., Gardner, W. L., \& Bernston, G. G. (1994). Bioelectrical echoes from evaluative categorizations: I. A late positive brain potential that varies as a function of trait negativity and extremity. Journal of Personality and Social Psychology, 67, 115-125.

Cacioppo, J. T., \& Gardner, W. L. (1999). Emotion. Annual Review of Psychology, 50, 191-214.

Center for the Study of Emotion and Attention. (1999). International affective picture system (IAPS): Technical manual and affective ratings. Gainesville, FL: NIMH-Center for the Study of Emotion and Attention, University of Florida.

Crites, S. L., Jr., Cacioppo, J. T., Gardner, W. L., \& Berntson, G. G. (1995). Bioelectrical echoes from evaluative categorization: II. A late positive brain potential that varies as a function of attitude registration rather than attitude report. Journal of Personality and Social Psychology, 68, 997-1013.

Cuthbert, B. N., Schupp, H. T., Bradley, M. M., Birbaumer, N., \& Lang, P. J. (2000). Brain potentials in affective picture processing: Covariation with autonomic arousal and affective report. Biological Psychology, 52, 95-111.

Dolan, R. J., Fletcher, P., Morris, J., Kapur, N., Deakin, J. F., \& Frith, C. D. (1996). Neural activation during covert processing of positive emotional facial expressions. Neuroimage, 4, 194-200.

Grave de Peralta Menendez, R., Hauk, O., Gonzalez Andino, S., Vogt, H., \& Michel, C. (1997). Linear inverse solutions with optimal resolution kernels applied to electromagnetic tomography. Human Brain Mapping, 5, 454-467.

Greenhouse, S. W., \& Geisser, S. (1959). On methods in the analysis of profile data. Psychometrika, 24, 95-112.

Hämäläinen, M., \& Ilmoniemi, R. (1984). Interpreting measured magnetic fields of the brain: Estimates of current distributions. Technical Report No. TKK-F-A559. Helsinki, Finland: Helsinki University of Technology.

Hamm, A. O., Greenwald, M. K., Bradley, M. M., \& Lang, P. J. (1993). Emotional learning, hedonic change, and the startle probe. Journal of Abnormal Psychology, 102, 453-465.

Hauk, O., Berg, P., Wienbruch, C., Rockstroh, B., \& Elbert, T. (1998). The minimum norm method as an effective mapping tool for MEG analysis. Paper presented at the Biomag98, Sendai, Japan.

Hauk, O., Keil, A., Elbert, T., \& Müller, M. M. (2002). Comparison of data transformation procedures to enhance topographical accuracy in time series analysis of the human EEG. Journal of Neuroscience Methods, $113,111-122$.

Hillyard, S. A., \& Anllo-Vento, L. (1998). Event-related brain potentials in the study of visual selective attention. Proceedings of the National Academy of Sciences USA, 95, 781-787.

Ilmoniemi, R. J. (1993). Models of source currents in the brain. Brain Topography, 5, 331-336.

Junghöfer, M., Bradley, M. M., Elbert, T. R., \& Lang, P. J. (2001). Fleeting images: A new look at early emotion discrimination. Psychophysiology, $38,175-178$.

Junghöfer, M., Elbert, T., Leiderer, P., Berg, P., \& Rockstroh, B. (1997). Mapping EEG-potentials on the surface of the brain: A strategy for uncovering cortical sources. Brain Topography, 9, 203-217.

Junghöfer, M., Elbert, T., Tucker, D. M., \& Rockstroh, B. (2000). Statistical control of artifacts in dense array EEG/MEG studies. Psychophysiology, 37, 523-532.

Kayser, J., Tenke, C., Nordby, H., Hammerborg, D., Hugdahl, K., \& Erdmann, G. (1997). Event-related potential (ERP) asymmetries to emotional stimuli in a visual half-field paradigm. Psychophysiology, 34, 414-426.

Keil, A., Müller, M. M., Gruber, T., Stolarova, M., Wienbruch, C., \&
Elbert, T. (2001). Effects of emotional arousal in the cerebral hemispheres: A study of oscillatory brain activity and event-related potentials. Clinical Neurophysiology, 112, 2057-2068.

Knosche, T., Praamstra, P., Stegeman, D., \& Peters, M. (1996). Linear estimation discriminates midline sources and a motor cortex contribution to the readiness potential. Electroencephalography and Clinical Neurophysiology, 99, 183-190.

Lang, P. J. (1980). Behavioral treatment and bio-behavioral assessment: Computer applications. In J. B. Sidowski, J. H. Johnson, \& T. A. Williams (Eds.), Technology in mental health care delivery systems (pp. 119-137). Norwood, NJ: Ablex Publishing.

Lang, P. J., Bradley, M. M., \& Cuthbert, B. N. (1990). Emotion, attention, and the startle reflex. Psychological Review, 97, 377-395.

Lang, P. J., Bradley, M. M., \& Cuthbert, B. N. (1997). Motivated attention: Affect, activation, and action. In P. J. Lang, R. F. Simons, \& M. T. Balaban (Eds.), Attention and orienting: Sensory and motivational processes (pp. 97-135). Hillsdale, NJ: Lawrence Erlbaum Associates.

Lang, P. J., Bradley, M. M., \& Cuthbert, B. N. (1999). International affective picture system (IAPS): Instruction manual and affective ratings. Technical Report A-4. Gainsville, FL: The Center for Research in Psychophysiology, University of Florida.

Lang, P. J., Bradley, M. M., Fitzsimmons, J. R., Cuthbert, B. N., Scott, J. D., Moulder, B., \& Nangia, V. (1998). Emotional arousal and activation of the visual cortex: an fMRI analysis. Psychophysiology, 35, 199-210.

Lang, P. J., Greenwald, M. K., Bradley, M. M., \& Hamm, A. O. (1993). Looking at pictures: Affective, facial, visceral, and behavioral reactions. Psychophysiology, 30, 261-273.

Laurian, S., Bader, M., Lanares, J., \& Oros, L. (1991). Topography of event-related potentials elicited by visual emotional stimuli. International Journal of Psychophysiology, 10, 231-238.

Luck, S. J., \& Hillyard, S. A. (1995). The role of attention in feature detection and conjunction discrimination: An electrophysiological analysis. International Journal of Neuroscience, 80, 281-297.

Lykken, D. T. (1972). Range correction applied to heart rate and to GSR data. Psychophysiology, 9, 373-379.

McCarthy, G., \& Wood, C. C. (1985). Scalp distributions of event-related potentials: An ambiguity associated with analysis of variance models. Electroencephalography and Clinical Neurophysiology, 62, 203-208.

Mini, A., Palomba, D., Angrilli, A., \& Bravi, S. (1996). Emotional information processing and visual evoked brain potentials. Perceptual and Motor Skills, 83, 143-152.

Müller, M. M., Keil, A., Gruber, T., \& Elbert, T. (1999). Processing of affective pictures modulates right-hemispheric gamma band EEG activity. Clinical Neurophysiology, 110, 1913-1920.

Palomba, D., Angrilli, A., \& Mini, A. (1997). Visual evoked potentials, heart rate responses and memory to emotional pictorial stimuli. International Journal of Psychophysiology, 27, 55-67.

Pascual-Marqui, R. D., Michel, C. M., \& Lehmann, D. (1994). Low resolution electromagnetic tomography: A new method for localizing electrical activity in the brain. International Journal of Psychophysiology, 18, 49-65.

Perrin, F., Pernier, J., Bertrand, O., Giard, M. H., \& Echallier, J. F. (1987). Mapping of scalp potentials by surface spline interpolation. Electroencephalography and Clinical Neurophysiology, 66, 75-81.

Pizzagalli, D., Regard, M., \& Lehmann, D. (1999). Rapid emotional face processing in the human right and left brain hemispheres: An ERP study. Neuroreport, 10, 2691-2698.

Schupp, H. T., Cuthbert, B. N., Bradley, M. M., Cacioppo, J. T., Ito, T., \& Lang, P. J. (2000). Affective picture processing: The late positive potential is modulated by motivational relevance. Psychophysiology, $37,257-261$.

Shagass, C. (1972). Electrical activity of the brain. In N. S. Greenbach \& R. A. Sternbach (Eds.), Handbook of psychophysiology (pp. 263-328). New York: Holt, Rinehart \& Winston.

Shagass, C., Amadeo, M., \& Roemer, R. A. (1976). Spatial distribution of potentials evoked by half-field pattern-reversal and pattern-onset stimuli. Electroencephalography and Clinical Neurophysiology, 41, 609-622.

Skrandies, W. (1995). Visual information processing: Topography of brain electrical activity. Biological Psychology, 40, 1-15.

Vogel, E. K., \& Luck, S. J. (2000). The visual N1 component as an index of a discrimination process. Psychophysiology, 37, 190-203.

(Received April 16, 2001; AcCePted March 19, 2002) 